\title{
Familial incidence and associated symptoms in a population of individuals with nonsyndromic craniosynostosis
}

\author{
Jaclyn Greenwood, MS, CGC'1, Pamela Flodman, MS, MSc, CGC1', Kathryn Osann, PhD, MPH², \\ Simeon A. Boyadjiev, $\mathrm{MD}^{3}$ and Virginia Kimonis, MD, MRCP ${ }^{1}$
}

\begin{abstract}
Purpose: Craniosynostosis is a common cranial malformation occurring in 1 per 2,000-2,500 births. Isolated defects (nonsyndromic) occur in $\sim 75 \%$ of cases and are thought to have multifactorial etiology. It is believed that each suture synostosis is a distinct disease, with varying phenotypes and recurrence rates.

Methods: We analyzed family histories of 660 mutation-negative nonsyndromic craniosynostosis patients and symptoms in 189 of these patients.

Results: The incidence rate of craniosynostosis was highest for firstdegree relatives of probands with metopic craniosynostosis (6.4\%), followed by those with complex craniosynostosis (4.9\%), sagittal craniosynostosis $(3.8 \%)$, lambdoid craniosynostosis $(3.9 \%)$, and coronal craniosynostosis $(0.7 \%)$. Across all suture types, siblings had a greater craniosynostosis incidence rate than parents (7.5 vs. $2.3 \%$ ). In phenotype comparisons, patients with complex craniosynostosis had
\end{abstract}

the highest frequency of reported symptoms and those with sagittal craniosynostosis had the lowest. Ear infections, palate abnormalities, and hearing problems were more common in complex craniosynostosis patients. Visual problems were more common in coronal craniosynostosis, and metopic craniosynostosis patients noted increased frequency of chronic cough.

Conclusion: Our data suggest that the genetic component of nonsyndromic craniosynostosis appears to be suture specific. The incidence rate of craniosynostosis among first-degree relatives varies by suture and family member. Additionally, the phenotype of each suture synostosis shows both unique and shared features.

Genet Med advance online publication 26 September 2013

Key Words: familial incidence; nonsyndromic craniosynostosis; symptoms

\section{INTRODUCTION}

Craniosynostosis (CS) is a common congenital anomaly resulting from the premature fusion of the cranial sutures, changing the skull's growth pattern. It is relatively common in the general population, occurring in 1 in 2,000-2,500 live births., CS can be classified into two broad categories, syndromic and nonsyndromic. Syndromic CS accounts for $25 \%$ of reported cases and can result from more than 180 identified monogenic syndromes. ${ }^{3,4}$ The remaining nonsyndromic/idiopathic cases are hypothesized to represent a multifactorial trait with genetic and environmental influences. ${ }^{1}$ Nonsyndromic CS can be subdivided on the basis of cranial suture involvement as sagittal, coronal, metopic, lambdoid, and complex types.

Sagittal CS is the most common type of nonsyndromic CS. It occurs in approximately 1 in 5,000 births and constitutes $40-55 \%$ of recognized nonsyndromic cases. ${ }^{5,6}$ It occurs predominantly in males at a male-to-female ratio of 3.5:1. ${ }^{7}$ Most of the cases occur sporadically, with $6 \%$ hypothesized to be familial. $^{7}$

Coronal synostosis is the second most common nonsyndromic CS, accounting for 20-25\% of cases and occurring in approximately 1 in 10,000 births. ${ }^{5,8}$ It can occur unilaterally (unicoronal CS) or bilaterally (bicoronal CS). Coronal synostosis occurs more frequently (60-75\% of cases) in females. ${ }^{9}$ Coronal CS is more likely to be associated with a known genetic syndrome or gene mutation. ${ }^{4}$ In addition, nonsyndromic coronal synostosis is believed to have a strong genetic influence. Studies have identified that $8-15 \%$ of nonsyndromic coronal synostosis patients have a positive family history. ${ }^{8,9}$

The incidence rate of metopic synostosis is approximately 1 in 15,000 births. ${ }^{1}$ It accounts for $\sim 14 \%$ of nonsyndromic CS cases and has a male-to-female ratio of 3.3:1.,10 Seventy percent of metopic synostosis patients have isolated occurrence with no other clinical malformations. ${ }^{10}$ Moreover, $5-10 \%$ of nonsyndromic metopic synostosis cases have a positive familial history. ${ }^{10,11}$

Lambdoid synostosis represents 3-5\% of nonsyndromic CS. ${ }^{5,12,13}$ It occurs in approximately 1 per 33,000 births. ${ }^{14}$ The genetics are largely unknown, and it is rare to have a positive family history with lambdoid synostosis. ${ }^{15}$

Five percent of the nonsyndromic CS cases involve multiple distinct sutures (two or more), also referred to as complex CS. ${ }^{5}$ Complex CS patients are observed to have an increased intracranial pressure in $67 \%$ of cases, and $75 \%$ of patients have a Chiari I malformation. ${ }^{16}$ The increased intracranial pressure often results in chronic headaches. In addition, there is a higher

${ }^{1}$ Department of Pediatrics, Division of Genetics and Metabolism, University of California, Irvine, Irvine, California, USA; ${ }^{2}$ Department of Medicine, Division of Hematology/ Oncology, University of California, Irvine, Irvine, California, USA; ${ }^{3}$ Department of Pediatrics, Section of Genetics, University of California, Davis, Davis, California, USA. Correspondence: Virginia E. Kimonis (vkimonis@uci.edu) or Simeon A. Boyadjiev (simeon.boyd@ucdmc.ucdavis.edu) 
incidence of developmental delay, and these patients are more likely to have CS resynostosis requiring additional surgeries. ${ }^{17}$

The precise cause, factors, and molecular mechanisms that cause nonsyndromic CS are not fully understood, although there is evidence that genetic factors play a role. A genetic role is suggested due to the higher rate of concordance in monozygotic compared with dizygotic twins (30 vs. $0 \%$ in sagittal nonsyndromic CS; and 43 vs. 5\%, in metopic CS) ${ }^{18}$ increased male-tofemale ratio in metopic and sagittal CS, ${ }^{7,10}$ and higher recurrence risk in affected families. ${ }^{19}$ Several environmental factors have also been associated with sagittal nonsyndromic CS, including parity, prematurity, intrauterine constraint, and maternal tobacco or nitrosatable drug use. ${ }^{20-23}$ In addition, more recently, a genomewide association study identified susceptibility loci near $B M P 2$ and $B B S 9$ that may influence sagittal CS development. ${ }^{24}$

The recurrence of nonsyndromic CS varies depending on the suture type and complexity. To date, only two families with lambdoid synostosis recurrence have been documented in the literature; therefore, this suture class is hypothesized to have a weaker genetic component. It has been previously hypothesized that there is a sibling recurrence risk of $2 \%$ for sagittal and metopic synostosis, $5 \%$ for unicoronal synostosis, and $10 \%$ for bicoronal and multisuture CS. ${ }^{7,910,25}$ However, these epidemiological studies predate advances in molecular diagnosis and include individuals in whom a gene mutation can now be identified. Therefore, further investigation using updated molecular testing information and classifications is important to provide families with more accurate assessments of risks.

Individuals with nonsyndromic CS can develop associated findings in addition to or secondary to CS. The published studies are limited due to their small sample sizes and absence of long-term follow-up. The associated symptoms that have been observed include increased rate of psychomotor delays, learning disabilities, visual defects, and maladaptive behaviors. ${ }^{26-31}$ How these symptoms are influenced by suture type is unclear.

The purpose of the present study was twofold. The first aim was to determine the incidence rate for first-degree relatives of patients diagnosed with suture-specific nonsyndromic CS. Second, we aimed to further investigate the associated symptoms in patients with suture-specific nonsyndromic CS. It is hypothesized that incidence rates and symptoms vary among suture types.

\section{MATERIALS AND METHODS}

This study is part of a larger study with many collaborating centers, collectively known as the International Craniosynostosis Consortium (ICC; https://genetics.ucdmc.ucdavis.edu/icc. $\mathrm{cfm}$ ), whose study center is at the University of California (UC), Davis.

The ICC database includes patients enrolled between 1993 and 2011. Informed consent was obtained for all patients. This study was approved by the institutional review boards of UC Davis and all participating institutions, including UC Irvine, and was conducted in accordance with institutional guidelines.
CS was documented by computerized skull tomography and/ or the protocol of the surgical correction. A clinical geneticist clinically assessed all patients, and patients with associated extracranial congenital anomalies or developmental delays were excluded.

Molecular analysis data were available through the ICC database. For patients without targeted molecular sequencing already performed as part of clinical management, hotspot-mutation analysis for FGFR1 exon IIIa, FGFR2 exons IIIa and IIIc, FGFR3 exon IIIa, and the entire coding sequence of TWIST was performed by DNA isolation, polymerase chain reaction amplification, and Sanger sequencing at UC Davis. Any sequence variation identified as being pathogenic was confirmed, documented, and entered in the comprehensive database.

The previously enrolled patients in the ICC were recontacted via e-mail or mail and invited to participate in a 20-minute follow-up survey. The survey asked patients or their parents to update their medical, family, and developmental histories. Those patients in the ICC database without e-mail or mailing addresses could not be recontacted.

Patients diagnosed with a genetic syndrome were excluded from analysis. In addition, patients with an identified mutation in FGFR1, FGFR2, FGFR3, or TWIST1 were also excluded and classified as syndromic. In addition, families with three generations of affected individuals were removed from analysis. Monozygotic or unknown-zygosity twins to the proband were excluded.

Results were grouped on the basis of suture diagnosis: sagittal, coronal, metopic, lambdoid, and complex. Individuals with a diagnosis of bilambdoid CS were grouped into the lambdoid category. Individuals with a diagnosis of bicoronal CS were grouped into the coronal category. All suture diagnoses were ascertained from the ICC database.

Lifetime incidence rates were estimated using the information available in the existing ICC database and the updated family history information ascertained from the follow-up survey. All incidence rates are expressed per 100 persons. Affected status of parents, full siblings, and children was available in the ICC database. For those who completed the update survey, their information was updated in the database to reflect the patient's current family structure and disease status. An incidence rate for affected parents, affected full siblings, and all first-degree relatives was calculated for each suture category and across all sutures. The observed incidence rate was calculated using the following formula:

$$
\text { Incidence rate }=\frac{\text { Number affected }}{\text { Total number }}
$$

Symptom data were collected from the ICC database of patients who responded to the follow-up survey. All patient symptoms were self- or parent-reported. The frequency of each symptom was calculated within each suture category. Patients with unspecified sutures were excluded from analysis. No statistical correction was made for multiple comparisons. 
Statistical analysis was conducted using SAS version 9.3 (SAS Institute, Cary, NC). Statistical significance was defined as a $P$ value $<0.05$. Pearson's $\chi^{2}$ test and Fisher exact analysis were used to calculate significance between groups. One-way analysis of variance was used to analyze the statistical associations across groups. A McNemar-Bowker statistical test was performed to estimate the significance of symmetry between the suture categories of the patient and the affected relative.

\section{RESULTS}

The ICC database contained 716 CS patients. Of these, 660 (92\%) met our inclusion criteria and comprised the primary study population. The excluded patients consisted of 53 with an identified syndrome or CS mutation and three with CS in three generations (consistent with an autosomal dominant etiology). A total of 197 enrolled patients (28\%) in the ICC database completed the follow-up survey. Of these, 189 (96\%) met our inclusion criteria and comprised the study population for associated symptoms. Demographic information by suture category is provided in Table 1 for the main study population $(n=660)$.

Patients were grouped into six suture categories: sagittal, coronal, metopic, lambdoid, complex, and unspecified. Forty-six percent of patients had sagittal CS $(n=301), 19 \%$ had coronal CS $(n=126), 15 \%$ had metopic CS $(n=99), 5 \%$ had lambdoid
CS $(n=31)$, and $11 \%$ had complex CS $(n=70) ; 5 \%$ reported an unspecified suture $(n=33$; Table 1$)$.

The mean age of the primary population at enrollment into the ICC database was 5.1 years (SD: 6.5); median age was 3.0 years. The unspecified suture group had the youngest median age (1.5 years), followed by the sagittal (2.9 years), coronal ( 3.0 years), complex (3.0 years), metopic (3.5 years), and lambdoid suture groups ( 4.7 years; Table 1$)$. Age did not vary significantly by suture category $(P=0.26)$.

Patients in the primary study population, across all suture categories except coronal, were more likely to be male. The male-to-female ratio across all suture types was 1.8:1, with the ratio of male-to-female patients in sagittal, coronal, metopic, lambdoid, complex, and unspecified suture types being $2.9,0.6$, 3.7, 6.8, 1.1, and 1.1, respectively (Table 1). The suture categories significantly varied by gender $(P<0.001)$.

Across all suture types, most of the patients identified themselves as Caucasian (71\%), with African Americans (2\%), Asians (2\%), Hispanics (2\%), and American Indians (1\%) also represented. A portion of the patients self-identified as other or mixed race/ethnicity (16\%), and another portion declined to specify their race/ethnicity (7\%; Table 1). African American, Asian, Hispanic, American Indian, and other racial categories were combined for this analysis, yielding a two-group

Table 1 Demographic information of craniosynostosis patients in the primary study population by suture category

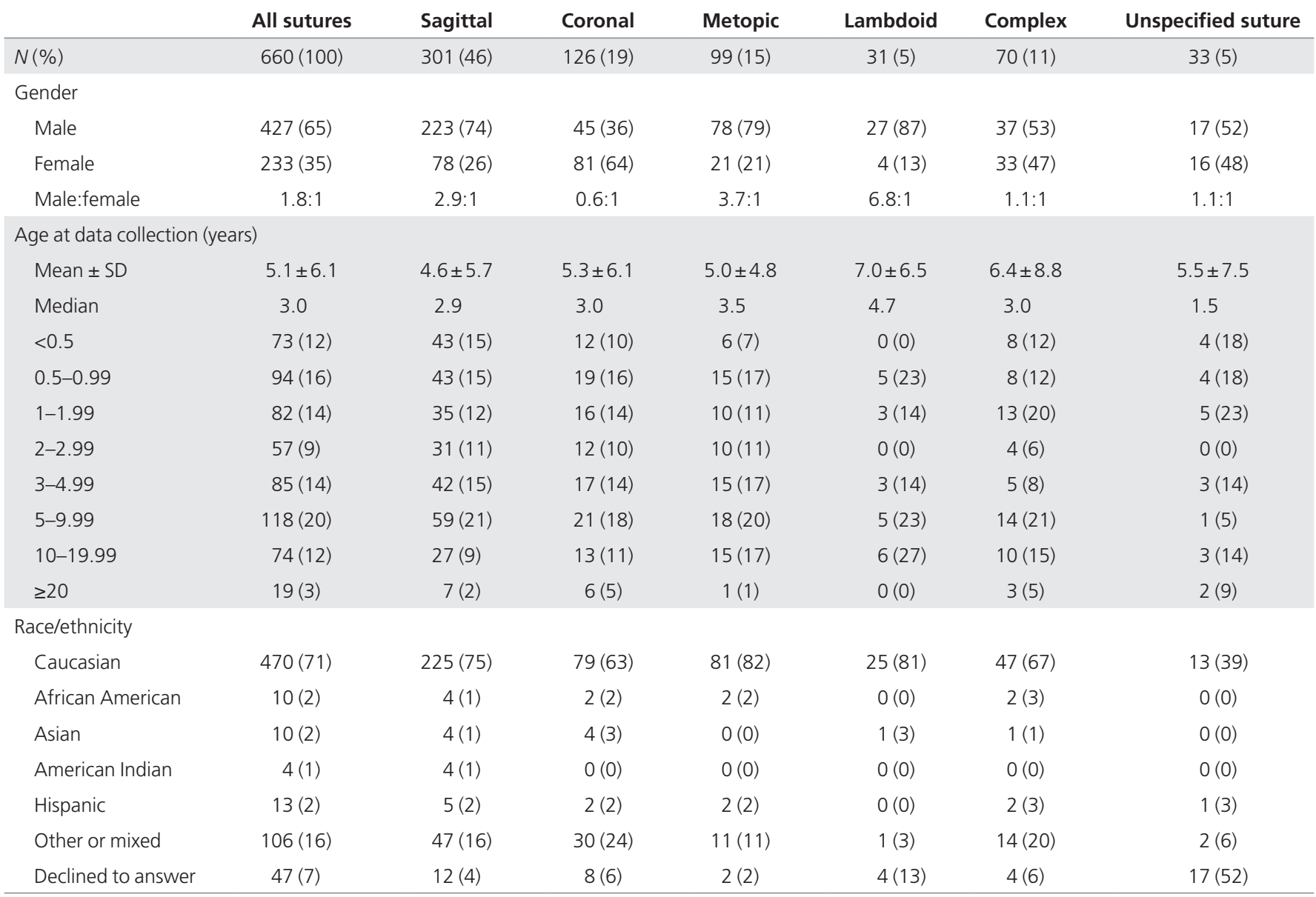


comparison (Caucasian and other). The suture categories significantly varied by Caucasian race $(P<0.001)$.

Among patients who responded to the survery, there was no difference by suture category $(P=0.674)$ or gender $(P=$ $0.38)$. However, the response rate did differ significantly by Caucasian ancestry $(P<0.001)$, with a higher response rate among Caucasians compared with non-Caucasians (35 vs. 15\%, $P<0.0001)$. The age distribution was also significantly different, with older participants responding to the survey, compared with those who did not respond $(P=0.0001)$. The mean age of responders at survey distribution was 9.0 years (SD: 6.7), and the mean age of nonresponders was 3.2 years (SD: 4.9).

\section{Lifetime incidence rates}

In our study, $7.3 \%$ of enrolled families had two or more firstdegree relatives affected with CS. The incidence rates are provided in Table 2 for all combinations of suture categories and by the affected relative's relationship to proband. The overall CS incidence rate for all suture types combined was 3.86 (reported per 100 patients). These rates were dependent on the suture type, in addition to the relationship and gender of the affected relative (Table 2 ).

The CS incidence rate was the highest for first-degree relatives of probands with metopic CS (6.4), followed by those with complex CS (4.9), sagittal CS (3.8), lambdoid CS (3.9), and coronal CS (0.7; Table 2). Across all suture types, siblings had a greater CS incidence rate than parents did (7.5 vs. 2.3 , respectively). In all suture categories except coronal and lambdoid types, male relatives had a higher incidence rate than female relatives. Overall, male relatives were more frequently diagnosed with CS than female relatives (4.9 vs. 2.9, respectively). The incidence rate among first-degree relatives did not differ significantly by gender of the proband.

The affected relative's suture category was analyzed for symmetry with the patient's suture category (Table 3 ). Sixty-eight percent of sagittal patients had relatives with sagittal CS $(n=$ 13), $55 \%$ of metopic patients had relatives with metopic CS ( $n=$ 6 ), and $29 \%$ of complex patients had relatives with complex CS $(n=2)$. There was no significant difference between the suture types of the relative and the patient $(P=0.988)$. Unspecified suture cases were excluded from analysis.

\section{Associated symptoms}

A total of 189 patients were included in the associated symptoms data set. They were grouped on the basis of their affected suture as sagittal, coronal, metopic, lambdoid, or complex type. The frequency of symptoms by suture category and $P$ values for each symptom across all suture categories are provided in Table 4.

Symptoms that significantly differed between suture categories include chronic fatigue $(P=0.02)$, chronic ear infections $(P=$ $0.03)$, hearing problems $(P=0.009)$, palate abnormalities $(P=$ $0.007)$, vision problems $(P<0.001)$, chronic cough $(P=0.03)$, incontinence $(P=0.003)$, and patient/parent-reported psychiatric concerns $(P=0.019)$. Sagittal CS had a significantly lower
Table 2 Observed lifetime incidence of craniosynostosis in relatives of probands with all sutures combined

\begin{tabular}{lccc}
$\begin{array}{l}\text { Relationship to } \\
\text { patient }\end{array}$ & $\begin{array}{c}\text { Observed } \\
\text { (no.) }\end{array}$ & $\begin{array}{c}\text { Total } \\
\text { population } \\
\text { (no.) }\end{array}$ & $\begin{array}{c}\text { Observed } \\
\text { incidence rate } \\
\text { (per 100) }\end{array}$ \\
\hline Parent & 23 & 1,022 & 2.25 \\
$\quad$ Mother & 9 & 538 & 1.67 \\
$\quad$ Father & 14 & 484 & 2.89 \\
Sibling & 34 & 456 & 7.46 \\
$\quad$ Male sibling & 20 & 213 & 9.39 \\
$\quad$ Female sibling & 14 & 243 & 5.76 \\
All first-degree & 57 & 1,478 & 3.86 \\
relatives & & & \\
Sagittal & & & \\
Parent & 12 & 496 & 2.42 \\
$\quad$ Mother & 3 & 264 & 1.14 \\
$\quad$ Father & 9 & 232 & 3.88 \\
Sibling & 14 & 192 & 7.29 \\
$\quad$ Male sibling & 10 & 98 & 10.20 \\
$\quad$ Female sibling & 4 & 94 & 4.26 \\
All first-degree & 26 & 688 & 3.78
\end{tabular}

relatives

$\begin{array}{lrrr}\text { Coronal } & & & \\ \text { Parent } & 0 & 195 & 0.00 \\ \quad \text { Mother } & 0 & 101 & 0.00 \\ \quad \text { Father } & 0 & 94 & 0.00 \\ \text { Sibling } & 2 & 89 & 2.25 \\ \quad \text { Male sibling } & 1 & 48 & 2.08 \\ \quad \text { Female sibling } & 1 & 41 & 2.44 \\ \text { All first-degree } & 2 & 284 & 0.70 \\ \text { relatives } & & & \\ \text { Metopic } & & & \\ \text { Parent } & 5 & 143 & 3.50 \\ \quad \text { Mother } & 2 & 75 & 2.67 \\ \quad \text { Father } & 3 & 68 & 4.41 \\ \text { Sibling } & 9 & 77 & 11.69 \\ \quad \text { Male sibling } & 5 & 28 & 17.86 \\ \quad \text { Female sibling } & 4 & 49 & 8.16 \\ \text { All first-degree } & 14 & 220 & 6.36\end{array}$

relatives

Lambdoid

Parent

$\begin{array}{llll}\text { Male sibling } & 0 & 12 & 0.00\end{array}$

$\begin{array}{llll}\text { Female sibling } & 1 & 14 & 7.14\end{array}$

$\begin{array}{llll}\text { All first-degree } & 3 & 78 & 3.85\end{array}$

relatives

Complex

Parent

Mother

Father

Sibling

Male sibling

Female sibling

All first-degree

relatives 
Table 3 Suture category of the patient by suture category of affected relative

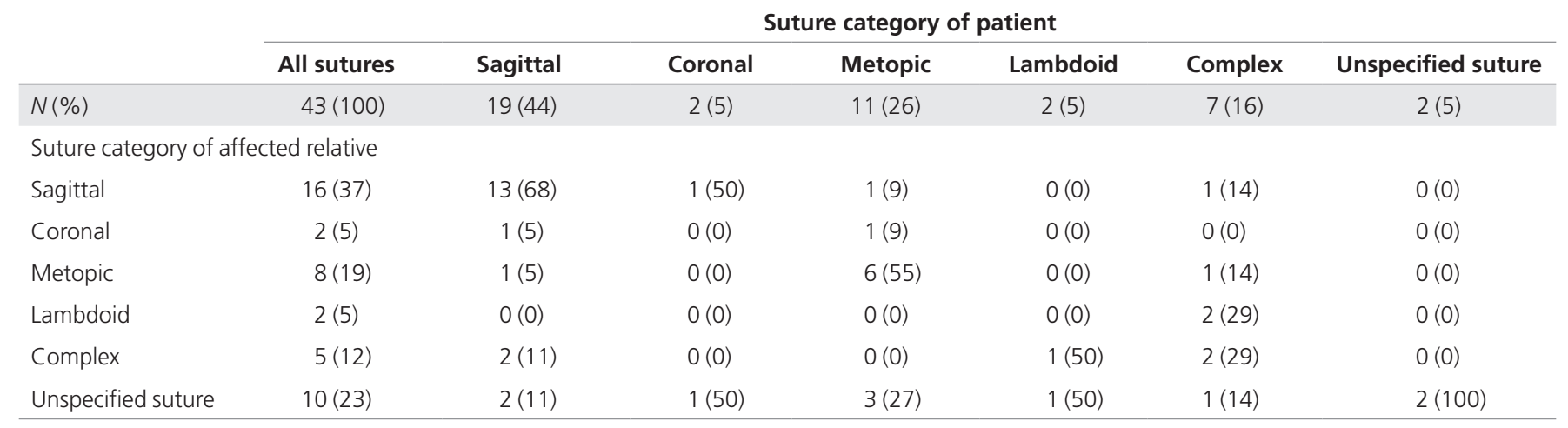

There was no evidence to reject the hypothesis of symmetry between patient's and affected relative's suture categories $(P=0.988)$. Unspecified suture cases were excluded from analysis.

portion of patients who reported chronic fatigue ( 2 vs. $13 \%, P=$ 0.02 ) and psychiatric concerns ( 0 vs. $6 \%, P=0.029)$ compared with other sutures. Coronal CS had the highest percentage of patients with vision problems compared with other sutures (45 vs. $18 \%, P=0.002$ ). Metopic CS had a significantly higher portion of patients who reported chronic cough compared with other sutures ( 22 vs. $4 \%, P=0.002$ ). Patients with complex CS were significantly more likely than those with other suture types to report chronic ear infections ( 43 vs. $20 \%, P=0.026$ ), hearing problems ( 29 vs. $5 \%, P=0.002$ ), palate abnormalities ( 24 vs. $4 \%$, $P=0.003$ ), or incontinence (14 vs. $0 \%, P=0.001$ ).

Across all sutures, each patient reported an average of three distinct symptoms (Table 5). There was a significant difference in the number of symptoms each patient reported by suture category $(P=0.018)$. Patients with complex CS reported, on average, the greatest number of symptoms (mean: 5.1) followed by those with coronal CS (mean: 4.2), metopic CS (mean: 3.9), lambdoid CS (mean: 3.1), and sagittal CS (mean: 2.5).

\section{DISCUSSION}

As expected, the sagittal suture was the most commonly affected suture, followed by coronal, metopic, complex, and lambdoid types. This pattern is consistent with that in the literature. ${ }^{7,9,10}$ Complex CS was more frequently observed in our study population (11\%) than previously reported (5\%).,12

The study population overall and for sagittal, metopic, and lambdoid CS types had a male majority, and the coronal CS group had a female majority. This finding agrees with previous published reports that observed a higher incidence rate of CS in males, particularly the sagittal and metopic CS types. ${ }^{7,10,32}$ This suggests that, overall, males are more susceptible to developing CS. Coronal CS has been found to occur predominantly in females. ${ }^{9,33}$ This indicates a different underlying etiology for coronal CS compared with other suture categories. There is limited research available on why this gender difference is consistently observed.

This study found a significant difference in Caucasian race by suture category. Metopic and lambdoid CS patients were more likely to be Caucasian, whereas coronal and complex CS patients were less likely to be Caucasian. No literature was found supporting this finding, and with our limited study group in some suture categories it is difficult to draw conclusions.

Three unrelated nonsyndromic CS patients had a striking family history of CS, with three generations of affected individuals. This suggests a yet-to-be-identified single-gene, autosomal dominant genetic etiology. It is possible that these families represent familial aggregation of a multifactorial condition, in a pattern that mimics autosomal dominant inheritance; the observed incidence rate of CS among first-degree relatives is slightly higher if these three families are included (4.23 vs. 3.86 per 100), but the overall conclusions do not change. Additionally, $6.8 \%$ of all nonsyndromic patients have a firstdegree relative affected with CS. These percentages are within the rate of 5-10\% suggested in previous studies. . $^{70,11}$

Sagittal, metopic, and lambdoid CS had a higher familial rate than that reported by previous studies. Previous studies predicted that $6 \%$ of patients with sagittal CS would have a positive family history of the disease, ${ }^{7}$ as opposed to our study's finding of $8 \%$. The percentage of metopic CS patients with a positive family history in our study was larger, at $12 \%$, than previously reported $(6 \%) .{ }^{10}$ Lambdoid CS, due to its rarity and limited case reports, was not previously believed to have a familial trend. We found that $6 \%$ of lambdoid CS patients had positive CS family history. These differences may be partially explained by ascertainment bias because families with more than one affected individual may be more likely to seek enrollment in research, although this study did not specifically seek to enroll multiplex families. In addition, our data are only as accurate as the family histories reported.

In all suture types combined, 3.9 per 100 of first-degree relatives of CS probands were reportedly diagnosed with CS. We suggest that family members of an affected patient have higher CS incidence rate compared with the general population frequency of $0.05 \%$. The observed incidence rate in relatives of patients with metopic CS was the highest among all the suture types (6.4), followed by those with complex CS (4.9), sagittal CS (4.3), lambdoid CS (3.8), and coronal CS (0.7). Previous studies predict patients with coronal and complex CS to have the 
Table 4 Associated symptoms by suture category

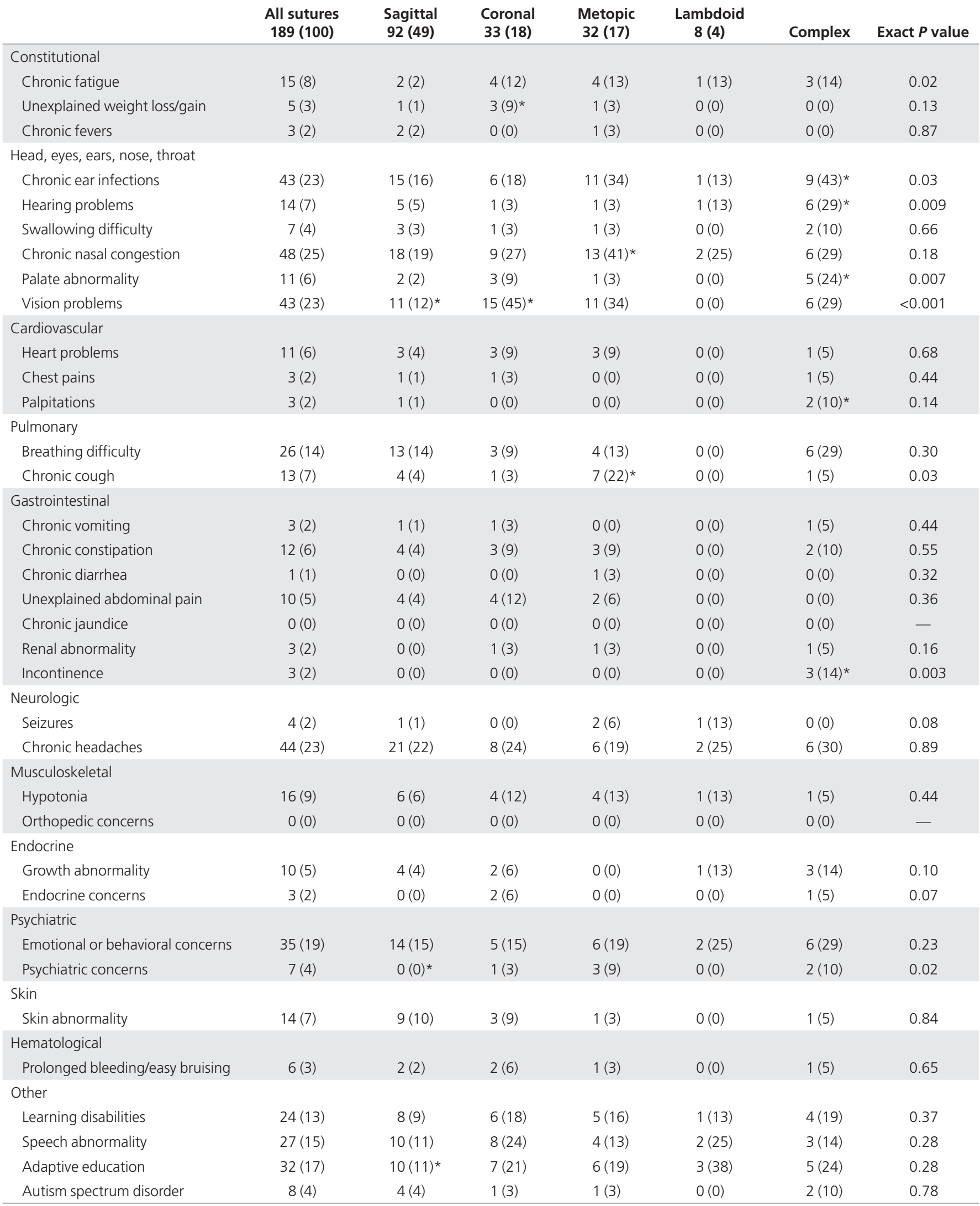

The values are given as $n(\%)$.

*Indicates significant $P$ values for specified suture type compared with all other suture types. 
Table 5 Mean number of distinct symptoms reported by patients by suture category

\begin{tabular}{cccccccc} 
& All sutures & Sagittal & Coronal & Metopic & Lambdoid & Complex & $\boldsymbol{P}$ value \\
\hline Mean number of symptoms reported \pm SD & $3.4 \pm 3.7$ & $2.5 \pm 2.9$ & $4.2 \pm 3.9$ & $3.9 \pm 4.0$ & $3.1 \pm 3.5$ & $5.1 \pm 5.2$ & 0.018 \\
\hline
\end{tabular}

highest rates among affected relatives and patients with metopic and sagittal to have the lowest rates. . $^{7,10,15}$

Further, $8-15 \%$ of patients with coronal CS were formerly found to have a positive family history. ${ }^{8,934}$ Coronal CS patients are the most likely CS category to have a known causative gene mutation or syndrome. ${ }^{12}$ In this study, $46 \%$ of excluded patients had coronal suture involvement. These patients had a diagnosed syndrome, a known gene mutation, or three generations of CS-affected individuals. Only $2 \%$ of coronal CS patients were identified to have affected relatives, most probably due to the exclusion criteria.

Our study did find a difference in incidence rate between genders of relatives and types of relatives. Overall, siblings had a higher CS incidence rate than parents did ( 7.5 vs. 2.3 per 100 patients). This difference in incidence rate between parents and siblings has not been identified in previously published literature and may be a reflection of ascertainment bias because families with more than one affected child may seek participation in research studies more than parents who are also affected. Alternatively, there may be a subset of the families in this study with sibling recurrence explained by autosomal recessive inheritance or germline mosaicism. To date, the autosomal recessive causes of CS include Baller-Gerold syndrome, Antley-Bixler syndrome, Opitz C syndrome, Lin-Gettig syndrome, IL11RA mutations, and Carpenter syndrome, all of which have many associated findings in addition to CS. ${ }^{35-37}$ Currently, little is known about the recessive genetic or germline mosaicism causes of nonsyndromic CS.

It is also likely that although some of the observed familial incidence rates can be explained by a recessive or dominant genetic etiology, others may be due to a multifactorial inheritance pattern. Many shared genes and environmental factors probably play a role in the development of nonsyndromic CS. ${ }^{20}$ More specifically, our data are consistent with a multifactorial threshold model in which males may have a lower threshold than females in sagittal, metopic, and lambdoid CS.

The gender of the CS patient's relative also shows an association with CS incidence rate. Overall, male relatives were more likely to be affected than female relatives for all suture categories combined (4.2 vs. 1.7 per 100 patients). As previously discussed, males appear to be more susceptible in developing sagittal, metopic, and lambdoid CS than females. Therefore, we would expect more male relatives to have these CS types than female relatives. It is predicted that familial incidence rate among female probands would be greater than that among male probands in this multifactorial threshold model. However, our study did not find a significant difference in incidence rates by proband gender.

The suture category of the affected patient did not differ significantly from the suture category of the affected relative.
Sixty-seven percent of metopic and 76\% of sagittal CS patients had the same suture CS as their relative. This finding of similar suture type in the affected patient and their affected relative supports the hypothesis that each suture synostosis has suturespecific genetic/environmental factors.

The second component of this study investigated the symptoms of CS patients. There was a significant difference in the number of symptoms patients reported by suture type. Sagittal CS patients reported the lowest average number of symptoms, whereas complex CS patients reported the greatest number. Complex CS patients have a higher incidence of ear infections, hearing problems, palate abnormalities, and incontinence than those in the other suture categories. This coincides with previous literature that found patients with complex CS to have the highest rate of complications compared with those patients with single suture CS. ${ }^{38}$ For some complex CS patients, multiple symptoms may suggest an undiagnosed syndrome. Sagittal CS patients had a lower incidence of fatigue, visual problems, psychiatric concerns, and need for adaptive education than the other suture categories. These patients did not have an increase in the incidence rate of any symptom when compared with the other suture categories. These findings suggest that sagittal CS is associated with the lowest number of complications and complex CS has the highest number.

Our study found coronal CS patients to be at the greatest increased risk for visual problems compared with all other suture categories ( 45 vs. $18 \%$ ). This finding is consistent with a previous report that found $45 \%$ of synostotic plagiocephaly (unilateral coronal CS) patients to have visual field abnormalities and an additional $10 \%$ of patients to report abnormal eye movements. ${ }^{28}$ An additional study observed an increase incidence of strabismus in a population of patients with unilateral coronal CS compared with the general population (58 vs. $4 \%$ ). ${ }^{39}$ This is thought to occur because the eye orbit is sometimes elevated on the affected suture side, thus affecting the visual field.

Learning disabilities and/or intellectual disability were reported less frequently in CS than expected but were more prevalent in CS patients than in the general population, by a difference of $9.7 \% .^{40}$ Our study found that, overall, 20\% of CS patients had some form of learning disability and/or intellectual disability. Previous studies had observed learning or cognitive disabilities at a $30-50 \%$ rate in nonsyndromic CS patients. ${ }^{26,27,40}$ Many learning disabilities or cognitive deficits are not diagnosed until children are observed to have difficulties in school. Thirtythree percent of our patients were less than 5 years of age and had yet to begin elementary school. However, this is unlikely to account for our lower-than-expected incidence rate because patients younger than 5 years did not have a significantly different incidence rate than those older than 5 years. Alternatively, our study only analyzed nonsyndromic CS patients, whereas 
other studies may not have used such strict exclusion criteria. Other studies may have included a significant proportion of patients that we excluded as syndromic, and the rate of learning disabilities may be higher in syndromic patients.

The strengths of this study include a well-characterized and large study population of patients with idiopathic CS. To date, this large-scale study is the first to analyze this specific population of CS patients. Limitations in this study include the possibility of unidentified ascertainment bias. Our study design may have favored reports from patients with more significant family history and/or clinical symptoms. In addition, the study includes multiple statistical comparisons, thereby increasing the probability that any specific statistically significant finding may have arisen by chance.

The results of this study support our hypothesis that firstdegree relatives of CS patients are at increased risk of CS as compared with the general population. Our study suggests that suture type, relationship, and the gender of the relative may have an effect on these risks. This information has the potential to be used in the genetic counseling setting to provide families with more current risk assessments. Additionally, our study also supported our hypothesis that the clinical symptoms can be influenced by suture diagnosis. Clinicians may choose to use this information to help determine appropriate medical evaluations and recommendations. However, this information should be used cautiously due to the possibility that these data may not accurately reflect the targeted patient population.

From the distinct and overlapping associations in our data, we suggest that the etiology of each suture synostosis has both unique and common underlying factors. Each suture CS represents a distinct disease and phenotype as supported by varying familial incidence rates and specific associated symptoms. However, there appears to be some shared mechanism between suture types as evidenced in families with relatives affected with different suture CS types. Future studies to identify the differing mechanisms of each suture category would provide further insight into overall recurrence risks and symptom management.

\section{ACKNOWLEDGMENTS}

We are thankful to all families who contributed to this study. We are indebted to Nisha Isaak, Christopher Nauta, Erica Goude, Linda Johnson, and Elijah Cherkez for patient recruitment. We thank John Graham, Joan Stoler, Pedro Sanchez-Lara, Jeffrey L. Marsh, Jayesh Panchal, James Boggan, Travis Tollefson, Craig Senders, Craig A. Vander Kolk, and George Jallo for contributing clinical information for this project. This work was supported by National Institute of Dental and Craniofacial Research/National Institutes of Health grants K23 DE00462, R03 DE016342, and R01 DE016886 to S.A.B. and in part by the Children's Miracle Network Endowed Chair. We thank the GCRC/ICTS at Boston Children's Hospital and UC Irvine.

\section{DISCLOSURE}

The authors declare no conflicts of interest.

\section{REFERENCES}

1. Cohen M, MacLean RE. Craniosynostosis: Diagnosis, Evaluation, and Management. 2nd ed. Oxford University Press: New York, NY, 2000:480.

2. Boulet SL, Rasmussen SA, Honein MA. A population-based study of craniosynostosis in metropolitan Atlanta, 1989-2003. Am J Med Genet A 2008;146A:984-991.

3. Wilkie AO, Bochukova EG, Hansen RM, et al. Clinical dividends from the molecular genetic diagnosis of craniosynostosis. Am J Med Genet A 2007;143A:1941-1949.

4. Kimonis V, Gold JA, Hoffman TL, Panchal J, Boyadjiev SA. Genetics of craniosynostosis. Semin Pediatr Neurol 2007;14:150-161.

5. Slater BJ, Lenton KA, Kwan MD, Gupta DM, Wan DC, Longaker MT. Cranial sutures: a brief review. Plast Reconstr Surg 2008;121:170e-178e.

6. Hunter AG, Rudd NL. Craniosynostosis. I. Sagittal synostosis: its genetics and associated clinical findings in 214 patients who lacked involvement of the coronal suture(s). Teratology 1976;14:185-193.

7. Lajeunie E, Le Merrer M, Bonaïti-Pellie C, Marchac D, Renier D. Genetic study of scaphocephaly. Am J Med Genet 1996;62:282-285.

8. Hunter AG, Rudd NL. Craniosynostosis. II. Coronal synostosis: its familial characteristics and associated clinical findings in 109 patients lacking bilateral polysyndactyly or syndactyly. Teratology 1977;15:301-309.

9. Lajeunie E, Le Merrer M, Bonaïti-Pellie C, Marchac D, Renier D. Genetic study of nonsyndromic coronal craniosynostosis. Am J Med Genet 1995;55:500-504.

10. Lajeunie E, Le Merrer M, Marchac D, Renier D. Syndromal and nonsyndromal primary trigonocephaly: analysis of a series of 237 patients. Am J Med Genet 1998;75:211-215.

11. Jehee FS, Johnson D, Alonso LG, et al. Molecular screening for microdeletions at 9p22-p24 and 11q23-q24 in a large cohort of patients with trigonocephaly. Clin Genet 2005;67:503-510.

12. Wilkie AO, Byren JC, Hurst JA, et al. Prevalence and complications of single-gene and chromosomal disorders in craniosynostosis. Pediatrics 2010;126:e391e400.

13. Huang MH, Gruss JS, Clarren SK, et al. The differential diagnosis of posterior plagiocephaly: true lambdoid synostosis versus positional molding. Plast Reconstr Surg 1996;98:765-74; discussion 775.

14. Rekate HL. Occipital plagiocephaly: a critical review of the literature. J Neurosurg 1998;89:24-30

15. Fryburg JS, Hwang V, Lin KY. Recurrent lambdoid synostosis within two families. Am J Med Genet 1995;58:262-266.

16. Renier D, Cinalli G, Lajeunie E, Arnaud E, Marchac D. [Oxycephaly, a severe craniosynostosis. Apropos of a series of 129 cases]. Arch Pediatr 1997;4:722729.

17. Chumas PD, Cinalli G, Arnaud E, Marchac D, Renier D. Classification of previously unclassified cases of craniosynostosis. J Neurosurg 1997;86: 177-181.

18. Lajeunie E, Crimmins DW, Arnaud E, Renier D. Genetic considerations in nonsyndromic midline craniosynostoses: a study of twins and their families. $J$ Neurosurg 2005; 103(suppl 4):353-356.

19. Boyadjiev SA; International Craniosynostosis Consortium. Genetic analysis of non-syndromic craniosynostosis. Orthod Craniofac Res 2007;10:129-137.

20. Sanchez-Lara PA, Carmichael SL, Graham JM Jr, et al.; National Birth Defects Prevention Study. Fetal constraint as a potential risk factor for craniosynostosis. Am J Med Genet A 2010;152A:394-400.

21. Zeiger JS, Beaty TH, Hetmanski JB, et al. Genetic and environmental risk factors for sagittal craniosynostosis. J Craniofac Surg 2002;13:602-606.

22. Gardner JS, Guyard-Boileau B, Alderman BW, Fernbach SK, Greene C, Mangione EJ. Maternal exposure to prescription and non-prescription pharmaceuticals or drugs of abuse and risk of craniosynostosis. Int J Epidemiol 1998;27:64-67.

23. Källén K. Maternal smoking and craniosynostosis. Teratology 1999;60:146150.

24. Justice CM, Yagnik G, Kim Y, et al. A genome-wide association study identifies susceptibility loci for nonsyndromic sagittal craniosynostosis near BMP2 and within BBS9. Nat Genet 2012;44:1360-1364

25. Carter CO, Till K, Fraser V, Coffey R. A family study of craniosynostosis, with probable recognition of a distinct syndrome. J Med Genet 1982;19:280-285.

26. Kapp-Simon KA. Mental development and learning disorders in children with single suture craniosynostosis. Cleft Palate Craniofac J 1998;35:197-203.

27. Kapp-Simon KA, Speltz ML, Cunningham ML, Patel PK, Tomita T. Neurodevelopment of children with single suture craniosynostosis: a review. Childs Nerv Syst 2007;23:269-281. 


\section{ORIGINAL RESEARCH ARTICLE}

28. Ricci $D$, Vasco $G$, Baranello $G$, et al. Visual function in infants with nonsyndromic craniosynostosis. Dev Med Child Neurol 2007;49:574-576.

29. Vasco G, Baranello G, Ricci D, et al. Longitudinal assessment of visual development in non-syndromic craniosynostosis: a 1-year pre- and post-surgical study. Arch Dis Child 2008:93:932-935.

30. Panchal J, Amirsheybani H, Gurwitch R, et al. Neurodevelopment in children with single-suture craniosynostosis and plagiocephaly without synostosis. Plastic Reconstructive Surg. 2001;108(6):1492-1498; discussion 1499-1500.

31. Becker DB, Petersen JD, Kane AA, Cradock MM, Pilgram TK, Marsh JL. Speech, cognitive, and behavioral outcomes in nonsyndromic craniosynostosis. Plast Reconstr Surg 2005:116:400-407.

32. Shahinian HK, Jaekle R, Suh RH, Jarrahy R, Aguilar VC, Soojian M. Obstetrical factors governing the etiopathogenesis of lambdoid synostosis. Am J Perinato 1998;15:281-286.

33. Kolar JC. An epidemiological study of nonsyndromal craniosynostoses. J Craniofac Surg 2011;22:47-49.
34. Mulliken JB, Gripp KW, Stolle CA, Steinberger D, Müller U. Molecular analysis of patients with synostotic frontal plagiocephaly (unilateral coronal synostosis). Plast Reconstr Surg 2004:113:1899-1909.

35. Panigrahi I. Craniosynostosis genetics: The mystery unfolds. Indian J Hum Genet 2011:17:48-53.

36. Nieminen P, Morgan NV, Fenwick AL, et al. Inactivation of IL11 signaling causes craniosynostosis, delayed tooth eruption, and supernumerary teeth. Am J Hum Genet 2011;89:67-81.

37. Hidestrand P, Vasconez H, Cottrill C. Carpenter syndrome. J Craniofac Surg 2009;20:254-256

38. Czerwinski M, Kolar JC, Fearon JA. Complex craniosynostosis. Plast Reconstr Surg 2011;128:955-961.

39. Macintosh C, Wall S, Leach C. Strabismus in unicoronal synostosis: ipsilateral or contralateral? J Craniofac Surg 2007;18:465-469.

40. Altarac M, Saroha E. Lifetime prevalence of learning disability among US children. Pediatrics 2007;119(suppl 1):S77-S83. 